\title{
Sensory Augmentation: Toward a Dialogue between the Arts and Sciences
}

\author{
Alwin de Rooij ${ }^{1}$, Michel van Dartel ${ }^{2}$, Antal Ruhl ${ }^{2}$, Hanna Schraffenberger ${ }^{1}$, Bente van \\ Melick $^{1}$, Mathijs Bontje ${ }^{1}$, Mischa Daams ${ }^{2}$ and Michel Witter ${ }^{2}$ \\ ${ }^{1}$ Tilburg University, Warandelaan 2, 5037 AB, Tilburg, NL \\ 2 Avans University of Applied Sciences, Parallelweg 21-24, 5223 AL, Den Bosch, NL \\ alwinderooijetilburguniversity.edu, mf.vandartel@avans.nl
}

\begin{abstract}
People sense the world by exploiting correlations between their physical actions and the changing sensory input that results from those actions. Interfaces that translate non-human sensor data to signals that are compatible with the human senses can therefore augment our abilities to make sense of the world. This insight has recently sparked an increase in projects that explore sensemaking and the creation of novel human experiences across scientific and artistic disciplines. However, there currently exists no constructive dialogue between artists and scientists that conduct research on this topic. In this position paper, we identify the theory and practice of sensory augmentation as a domain that could benefit from such a dialogue. We argue that artistic and scientific methods can complement each other within research on sensory augmentation and identify six thematic starting points for a dialogue between the arts and sciences. We conducted a case study to explore these conjectures, in which we instigated such a dialogue on a small scale. The case study revealed that the six themes we identified as relevant for a dialogue on sensory augmentation emerge rather spontaneously in such a dialogue and that such an exchange may facilitate progress on questions that are central to the theory and practice of sensory augmentation. Overall, this position paper contributes preliminary evidence for the potential of, and a starting point for, a dialogue between the arts and sciences that advances our understanding of sensory augmentation and the development of applications that involve it.
\end{abstract}

Keywords: Art-Science Collaboration; Cognitive Science; Interfaces; New Media Art; Sensory Augmentation; Sensory Substitution.

\section{Introduction}

"Art being a thing of the mind, it follows that any scientific study of art will be psychology", states Friedlander in Gombrich's book Art and illusion [1] (p. 3). In the dialogue between the arts and psychology that Gombrich advocates however, art plays a mere instrumental role in the development of theory on perception. For instance, pictorial artworks are discussed as support for a representational theory of perception, yet such theory is not explicitly considered to hold any value for the creation of art. It has been shown that theory of perception can however also advance the field of art. In turn, 
artistic work that is grounded in such theory can be relevant to the study of perception [2]. In this position paper, we argue that this especially goes for research that considers perception as action (i.e., the sensorimotor coordination approach [3]), rather than a passive processing of symbols (e.g., computational approaches to perception [4]).

Theory that considers perception as an activity suggests that people make sense of the world by exploiting the correlations between their physical actions and the changing sensory input that results from those actions, i.e. sensorimotor contingencies [5]. Parallel to the emergence of such scientific theory, recent art theory suggests that art is no longer something to look at or listen to from a distance, but a call to action that challenges us to understand or make sense of something [6], and that art practices can be considered modes of thought based on acts of creation [7]. As perception research shifts to studying the correlations between sensory input and physical actions, art is shifting focus to intervening and subverting such active relationships between humans and their surroundings [8]. This suggests that a dialogue on perception research between artists and scientists is possible now more than ever. Besides novel scientific insight inspiring new artistic experiences, such a dialogue could also be mutually constructive because "in the sciences [...] the subjectivity that accompanies experience is usually seen as an undesired variable that is to be controlled rather than enhanced" whereas "creative research practices emphasise the role of personal or subjective experiences" [9] (p. 90). Thus, where science aims to understand subjective phenomena, such as perception, attempts to rule out rather than enhance the 'undesired variable' of subjectivity can be in the way of progress. Therefore, the arts and sciences can complement each other, yet need to be desegregated. In what follows, we identify the art and science of sensory augmentation, identify six themes as point of departure for a dialogue between the arts and sciences, and present a small case study on what this dialogue could bring about.

\section{Sensory augmentation in the arts and sciences}

Interfaces that translate non-human sensor data to signals that are compatible with the human senses can augment our abilities to make sense of the world, and are used across the sciences and the arts $[2,10]$. One fundamental experiment to the field of sensory augmentation has resulted in both acclaimed artistic and much-debated scientific outcomes; a class of experiments often referred to as 'inverted vision'. In these experiments, goggles with mirrors that alter the visual consequences of moving one's eyes, e.g. reversing up and down, are typically used. While experiments by scientists Remy Stratton [11] and Ivo Kohler [12] are often taken as empirical proof for active approaches to perception [3], and continue to inspire research on the topic (e.g., [13]), these experiments also had their impact on the arts. Artist Carsten Höller, for instance, has extensively researched the application of 'inverted vision' in aesthetic experiences, pioneering a novel type of active relationship between an artwork and its audience [8].

Sensory substitution goes one step further in intervening and subverting correlations between the sensory changes that result from the movement of our sensors. It takes the sensory changes of one (artificial) modality and correlates these to sensory changes in another. By doing this consistently, the sensory correlations that we are accustomed to 
within one modality transfer to another and augment the original sensory experience. A classic example is a series of experiments by Bach-y-Rita [14] for which he developed an interface that substituted visual information into tactile stimulation by mapping a low-res camera image onto a grid of solenoids worn on the stomach or back. After training, subjects reported the experience of 'seeing with the skin'. This work demonstrates the possibility of developing interfaces to establish novel sensorimotor relationships, through which new contingencies can be mastered to make sense of and experience the world; experiences otherwise non-existent. Although sensory augmentation has since been widely explored scientifically (see, e.g., $[10,15])$, the potential initially projected on Bach-y-Rita's breakthrough findings has yet to be fully realized in both art and science. First, laboratory studies have shown that learning sensorimotor contingencies follows generalisable lawful patterns [16], but reports of participant's having novel subjective sensory experiences during such studies, such as "seeing with the skin", are mostly anecdotal [17]. Second, laboratory studies into sensory augmentation have been argued to neglect testing users for "novel kinds of stimuli [...] for which they lack pre-existing knowledge" [18], which omission draws into question whether sensory augmentation interfaces can elicit truly novel sensory experiences. We argue that investigation of such novel experiences (i) calls for a broad imagination on what such "novel kinds of stimuli" [18] could entail, and (ii) requires the creation of personal subjective experiences and modes of research that allow for unavoidable subjective and personal experiences (see also [19]). Such imagination, as well as the creation of personal subjective experiences, are key to the domain of art $[2,6,8,9]$. This suggests that the generalisability that comes with the scientific method can help develop new theory, on the basis of which new subjective experiences can be designed. Reversely, artistic exploration of subjective experiences can form a basis to advance theory. Therefore, we propose an integrated approach to advance the theory and practice of sensory augmentation based on the instigation of a dialogue between art and science on the topic.

\section{Themes for a dialogue between the arts and sciences}

To facilitate the envisioned dialogue between art and science we develop six themes that emerged consistently in discussions between the authors of this paper and that can be used to structure the envisioned dialogue in a manner that advances both domains.

\subsection{Understanding sensory augmentation}

Three themes for the envisioned dialogue emerged that may advance our theoretical understanding of sensory augmentation: (i) apparatuses, (ii) mapping, and (iii) contingency learning.

First, the apparatuses that artistic and scientific projects use to effectively achieve sensory augmentation should be a theme in the envisioned dialogue on sensory augmentation. Where scientists typically develop apparatuses to map sensor data to human senses, e.g. Bach-y-Rita's TVSS interface [16], artists may conceive completely new types of senses or enable the body's senses in unlikely places [2]. An example of the 
latter is the Blind Smell Stick by artist Peter De Cupere, which extends the olfactory sense via a long rigid tube that hovers just above the ground, sucks in air, and blows it into the user's nose, providing a novel way of navigating and experiencing cities [21]. Scientific apparatuses for sensory augmentation, such as Bach-y-Rita's TVSS interface, as well as artistic apparatuses, such as De Cupere's Smell Stick, help us to understand human experience better and inspire new research. While hitherto such pieces of equipment predominantly inspired new research within the domain within which they were conceived, it seems plausible that scientific analysis of the aesthetic experience of sensing at a distance, as brought about by the Smell Stick, may advance theory of perception, while artistic analysis of TVSS interfaces may just as well advance theory of aesthetics.

Second, effective and meaningful sensory augmentation also depends on how the mapping from an (artificial) sensor to a human sense is designed [20]. For instance, [19] showed that speech-to-touch mapping leads to signal loss because speech is sensed at a dimensionality that exceeds the actuation capabilities of a haptic interface. However, mapping speech signals to movement patterns, rather than to static touch points on the skin, increased the ability of people to accurately classify the content of the speech signals. Artistic work has a long history of evoking experiences associated with one sense by using another $[22,23,24,25]$. In doing so, it provides worthwhile leads for developing new mappings, which can enable effective sensory augmentation.

Third, the meaning and richness of the experiences that sensory augmentation elicits also depends on the context in which contingency learning is achieved. Where scientists typically use operant conditioning in a lab context with limited success [16, 20], artists pioneered contingency learning through narratives [2] and "in the wild" [7]. In one of Carsten Höller's artworks, for instance, participants wore inverted-vision goggles on a rooftop terrace with a spectacular view over London. After a brief period of adaptation, some relatively easily managed to walk to the edge of the terrace, to be rewarded with a reversed view over London. Interestingly, the period of adaptation to the inversion was characterized by social play and 'having fun' [8]; signaling their importance in learning sensory contingencies. Thus, a dialogue about contingency learning may help to understand how sensory augmentation can achieve rich and meaningful experiences.

\subsection{Applying sensory augmentation}

Three other themes for the envisioned dialogue emerged that may advance the practical application of sensory augmentation: (i) test environments, (ii) new application domains, and (iii) commercial potential.

First, developing effective sensory augmentations can benefit from a dialogue about test environments. Ideally, these enable user-testing to optimise the way sensory augmentation is achieved. Virtual reality (VR) may be particularly suitable as a starting point due to VR's psychological realism [26, 27], ability to simulate interfaces and environments [28], and shared history in art and science [27]. For example, simulated variations on distance-to-audio mappings have been tested in a virtual maze to study what mappings lead people to navigate through the maze the quickest [28]. The same flexibility may help adapt scenarios and narratives to pre-test how to achieve rich and meaningful immersive sensory experiences effectively. 
Second, utilising the questions that artists and scientists tend to ask can help to explore new application domains. Art is often driven by 'What if?' questions, while science explores 'How?' questions $[2,18]$. Conveying the experience of a sensory augmentation and asking 'What if?' can reveal its utility, complementing the need to understand 'How' it works. This way, new applications can be probed to see whether (further) research and development is justifiable. Illustrative of such new domains is augmented navigation. For example, [29] studied how a tactile-vision navigational aid could support the visually impaired. A similar interface was used by [30] to suggest spatial dimensions to an audience by exploring their ability to use such augmentations and how this impacted their experiences. These findings suggest that combining art and science can support the exploration of new application domains.

Third, artists have set up 'spoof' companies to probe the commercial potential of innovative products, including sensory augmentation interfaces. For instance, 'Eye Candy', a lollipop-like device inspired by Bach-y-Rita's BrainPort tongue interface, was marketed online as an off-the-shelf product by Eye Candy Can Ltd. [31]. The product was 'released' in six different flavours; the 'FOCUS' flavor, for instance, promised a tactile sense of "Direction giving arrows that help to improve focus and attention". Approximately 68.000 people attempted to purchase 100.000 lollipops [2], yielding the online venture a commercial success. Such artistic work can gauge what kinds of sensory augmentation are commercially viable and may help guide the development of sensory augmentation interfaces in the direction of their commercial potential.

\section{Case study: A sensory augmentation masterclass}

We conducted a small case study to confirm whether or not the selected themes could form a constructive starting point for the envisioned dialogue and to gather preliminary evidence for its potential. To instigated a dialogue between artists and scientists we organised a masterclass on sensory augmentation in which they explored sensory augmentation interfaces that are commonly used in scientific and artistic research practices.

Participants. Besides two session leaders, 13 early to mid-career professionals participated in the study; an architect, writer, curator, material scientist, stylist, two cognitive psychologists and two media artists and three media designers. The masterclass was held during the STRP Biennale in Eindhoven (NL) on March 28, 2017.

Masterclass. The masterclass was structured on the basis of Wallas' four stage model [31]. First, participants engaged in two preparatory activities: (i) an introduction by the session leaders to the art and science of sensory augmentation, which included a discussion of the six themes introduced above, and (ii) a demonstration session in which four participating researchers demonstrated four sensory augmentation interfaces (Fig. 1): (i) Inverted-vision goggles. Five goggles with prisms that alter the visual consequences of moving one's eyes, reversing up and down (from [33]); (ii) Pupil size-auditory feedback interface. An eye tracker with software that maps pupil size to sound; (iii) Haptic vision interface. An interface that maps camera images to the tactile sense via a grid of solenoids; and (iv) Magnetic north interface. A vibrating belt that indicates the direction of the magnetic north (from [16]). These demonstrations were followed 
by the formation of breakout groups that each explored one or two of the interfaces. Each demonstrator chaired a breakout group and the remainder of the participants were distributed over the groups, on the basis of their individual preference. The two smallest groups, with those who selected the inversion goggles or haptic vision interface, were merged together to create groups of similar sizes. Using the interfaces as discussion starters enabled participants to first-hand explore how such interfaces can augment our experience of the world. After being given ample time to explore the sensory interfaces and share initial thoughts amongst the group, participants took a lunch break of approximately $45 \mathrm{~min}$. This enabled them to replenish and incubate, benefitting subsequent idea generation [33]. Third, the groups purposefully explored the interfaces to elicit ideas on (i) new research questions and (ii) new concepts and prototypes that could lead to insight into these questions. Fourth, the questions, concepts, and prototypes were presented, demonstrated, and discussed in a plenary session. This allowed for verification and reflection of the research questions, concepts, and prototypes developed.

Documentation. The masterclass was documented photographically throughout the day. The plenary closing session was also filmed. On the basis of this documentation we were able to cite several key observations about the research questions and concepts developed in the masterclass, which we will discuss in the section below.

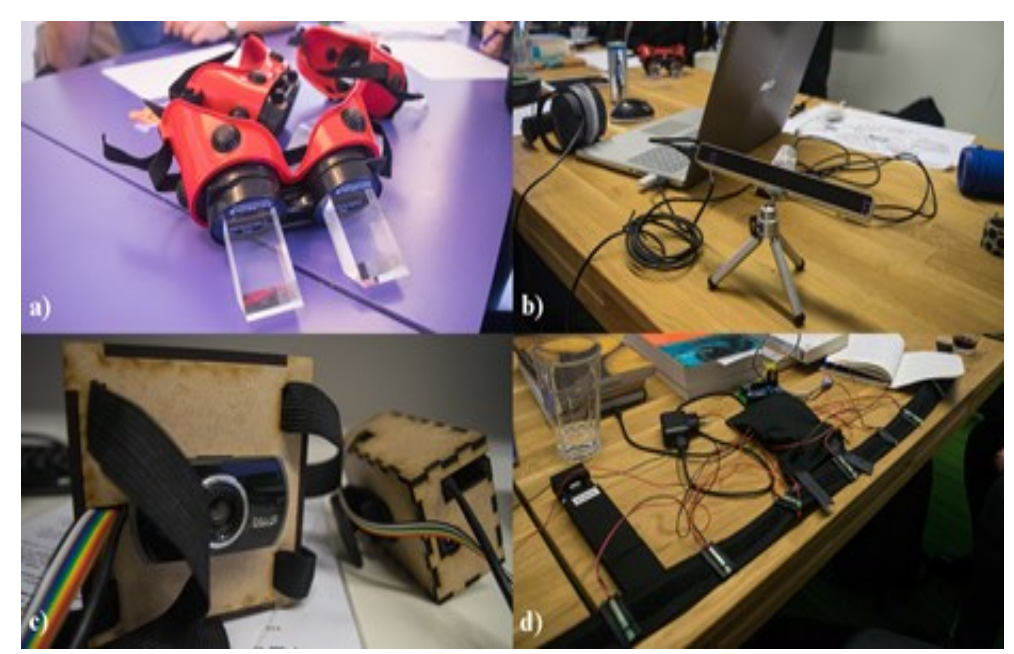

Fig. 1. The sensory augmentation interfaces used in the study: a) Inverted vision goggles, b) pupil size-auditory feedback interface, c) haptic vision interface, d) magnetic north interface. (Photos by Stanley Obobogo Badoana)

\section{$5 \quad$ What did the case study reveal?}

The masterclass resulted in three new research questions and concepts that fitted the previously selected themes, and touched upon the creation of novel subjective experiences and the development of novel kinds of stimuli (section 5.1). It also led to general observations regarding the dialogue it instigated, which may prove useful leads for future work toward the envisioned dialogue between the arts and sciences (section 5.2). 


\subsection{New research questions and concepts}

Three new research questions and concepts resulted from the masterclass:

(i) Haptic Vision: Using haptic augmentation to support inverted vision. The group that explored the inversion goggles and haptic vision interface conceived and developed the research question: "Can one measure the effects of sensory substitution using an inverted vision task?", investigating the themes contingency learning and test environments. The participants created a game in which two participants compete to trace a zigzagged line as fast as possible, from top to bottom with one hand. Both contesters wear inverted vision goggles, yet one of them also wears the haptic vision interface on the hand with which the line is traced. The contester that first reaches the bottom of the line wins. Playing the game led to several interesting observations: (i) Re-learning hand-eye coordination under inverted vision can benefit from haptic-vision substitution support when it is used strategically, e.g. as error feedback (i.e., "..checking if I am still on the line.", as one contestant called it); (ii) Strong reliance on the haptic interface can also interfere with attempts to overcome disorientation (e.g. "The haptics provide too much input", one contestant yelled out to his competitor); (iii) even within the limited time of the closing session, repeated performance seemed to suggest a learning curve for contestants wearing the haptic device, which might indicate that participants improved their ability to interpret the distorted visual sense and haptic information simultaneously; and (iv) a hybrid interface, which combines sensory substitution with other forms of augmentation, points in interesting directions for future research. The range of fundamental questions that were elicited during the short plenary presentation of this outcome, attests to the usefulness of allowing and enhancing personal and subjective experiences in sensory augmentation research.

(ii) The pupil DJ: Emotion regulation by sensing pupil size. The group that explored the pupil size-auditory feedback interface conceived and developed the research question: "Can sensing pupil size be used to regulate emotion?" To this end, the group touched upon the apparatuses theme. The interface was originally designed to map pupil size to sound, but after some exploration of the interface the group decided to focus on the link between pupil size and emotion instead. Based on the assumption that pupil size correlates with emotion, the group investigated the link between music and emotion, for which the interface was slightly adapted. Initial testing showed that pupil size indeed varies between songs, and that some changes roughly correspond to the emotional reaction to a song. The group subsequently discussed the potential of selecting and playing songs on the basis of pupil size, as a means to regulate emotion, and speculated on a novel apparatus to explore whether meaningful relationships could emerge from a sensorimotor loop between pupil size and music selection. With this, they alluded to pupil behaviours made perceptible as novel stimuli that enable an added sense of one's own emotions; and suggest a new application domain for sensory augmentation based on pupil size.

(iii) Spouse detector: A novel sense for the (un)faithful. The group that explored the magnetic north interface conceived and developed the research question: "Can a belt that vibrates in the direction of your spouse support a relationship?". The interface was 
conceptualised as providing location awareness of one's partner, rather than the magnetic north. In this concept, a set of mappings, i.e. vibration patterns, provide a sense of objective support that "augments the ability to connect and stay faithful to a romantic partner, even in his or her absence", as the group described it. Vibration patterns were elaborated to establish a sense of the other's presence and potentially tangibly convey their emotional states. The concept led to constructive discussions on (i) the usefulness of augmenting such directional awareness with a tactile representation of the other's inner rhythms, e.g. breathing or heartbeats, and (ii) whether people could learn to interpret such patterns as meaningful information about the other or should rely on algorithms to classify such information for them. By exploring novel stimuli, tuned to the consequences of one's actions for romantic relationships, this concept illustrates how the dialogue we envision may break open unexpected new application domains for sensory augmentation interfaces.

\subsection{General observations}

Although each group followed its own unique trajectory towards the outcomes described above, four general observations can be made regarding their dialogues.

The preparatory activities gave rise to highly divergent discussions in each breakout group. Although the interfaces and demonstrator's practices were provided as a starting point for a dialogue, these almost instantly diverged in very different directions. Two general observations can be made regarding the dialogues overheard during this phase of the masterclass: First, discussions commonly veered towards the practices of the participants; and second, each group displayed a tendency towards thought experiments in which the interface is applied to everyday situations, such as to listening to music or to maintaining romantic relationships. This may indicate that 'the everyday' makes fruitful common ground for researchers to explore when individual backgrounds vary.

After the replenishment and incubation during the break, it took each group relatively long to transition towards idea generation. In fact, most of the two hours reserved after the break to converge towards a research question and concept, was consumed by exploring multiple ideas in parallel or by diverging even more by formulating yet more new ideas. Only when the session leaders announced that each group had 5 minutes left to finalise their presentations, each group rapidly converged by abandoning secondary ideas or combining several ideas into one presentable outcome. The importance of time pressure for convergence [34], can therefore be added as a third general observation.

The verification and reflection that followed during the closing plenary session revealed that the research questions, concepts, and prototypes (i) matched several of the themes identified in section 3, and (ii) supports the arguments developed in sections 1 and 2. That is, the range of observations made during the case study attest to the potential of allowing and even enhancing otherwise confounding variables, such as those that associate with personal and subjective experience, and at least two outcomes alluded to the creation of novel kinds of stimuli; relative location to a spouse or audio-feedback based on pupil size. Thus, a fourth observation can be added; the study's results attest to the potential of a dialogue between the arts and sciences about sensory augmentation. 


\section{Conclusion}

In this position paper, we argued that a dialogue between the arts and sciences may advance our understanding of sensory augmentation and can be useful for developing novel sensory augmentation applications. As a starting point we introduced six themes, around which this dialogue could be structured, and presented a small case study in which a dialogue between artists and scientists was initiated. The study yielded several new research questions and concepts that (i) indicate how informative personal and subjective experiences can be, strengthening our claim that a dialogue between art and science may help overcome the scientific limitation that the subjective is generally considered an 'undesired variable' (sections 1 and 2); (ii) alluded to novel kinds of stimuli, suggesting that a dialogue between art and science can indeed benefit the broad imagination needed to conceive the "novel kinds of stimuli" for which we lack "pre-existing knowledge" [18] that is required to advance the field (section 2); and (iii) the majority of the described themes emerged during the dialogue, supporting the use of the selected themes as a starting point for the envisioned dialogue (section 3). Thus, this paper contributes preliminary evidence for the potential of, and a starting point for, a dialogue between the arts and sciences that advances our understanding of sensory augmentation and the development of applications that involve it.

\section{References}

1. Gombrich, E.H.: Art and illusion. Phaidon, London (1977).

2. Schwartzman, M.: See yourself sensing: redefining human perception. Black Dog Publishing, London (2011).

3. Noë, A.: Action in Perception. MIT Press, Cambridge, MA (2004).

4. Marr, D.: Vision. W.H. Freeman, San Francisco (1982).

5. O'Regan, J.K., \& Noë, A.: A sensorimotor account of vision and visual consciousness. Behavioral and Brain Sciences 24(5), 883-917 (2001).

6. Noë, A. Strange Tools. Art and Human Nature. Hill and Wang, New York (2015).

7. Manning, E. \& Massumi, B.: Thought in the Act. University of Minnesota Press, Minneapolis, MN (2014).

8. Van Dartel, M.F.: Aesthetics in the Wild: Art and Design Practices and Pedagogies after the Situated Turn. Avans, Breda (2016).

9. Biggs, M. \& Karlsson, H.: Research in the Arts. Routledge, New York, NY (2012).

10. Froese, T., McGann, M., Bigge, W., Spiers, A. \& Seth, A.K. 2012. The enactive torch: a new tool for the science of perception. IEEE Transactions on Haptics 5, 4, 365-375.

11. Stratton, G.M.: Vision without inversion of the retinal image. Psychological Review 4, 463481 (1897).

12. Kohler, I.: The formation and transformation of the perceptual world, volume 3 of Psychological issues. International university press, monograph 12 (1964).

13. Miyauchi, S., et al.: Adaptation to left-right reversed vision rapidly activates ipsilateral visual cortex in humans. Journal of Physiology Paris 98, 207-219 (2004). 
14. Bach-y-Rita, P., Collins, C.C., Saunders, S.A., White, B. \& Scadden, L.: Vision substitution by tactile image projection. Nature 221, 963-964 (1969).

15. Kercel, S.W. \& Bach-y-Rita, P.: Noninvasive coupling of electronically generated data into the human nervous system. In: Wiley Encyclopedia of Biomedical Engineering, Akay, M., Ed., Wiley, 1960-1974 (2006).

16. Kaspar, K., König, S., Schwandt, J. \& König, P.: The experience of new sensorimotor contingencies by sensory augmentation. Consciousness and Cognition 28, 47-63 (2014).

17. Ward, J \& Meijer, P.: Visual experiences in the blind induced by an auditory sensory substitution device. Consciousness and Cognition 19, $492-500$ (2010).

18. Deroy, O. \& Auvray, M. (2012). Reading the World through the Skin and Ears: A New Perspective on Sensory Substitution. Frontiers in Psychology 3.

19. Sha, X.W.: Poiesis and Enchantment in Topological Matter. Cambridge: MIT Press (2013).

20. Novich, N.: Sound-to-touch sensory substitution and beyond. PhD thesis. Rice University (2015).

21. De Cupere, P.: Blind smell stick. Retrieved 02-11-2016 from http://www.blindsmellstick.com/index.php/about (2012).

22. De Rooij, A., Broekens, J. \& Lamers, M.H.: Abstract expressions of affect. International Journal of Synthetic Emotions 4, 1, 1-31 (2013).

23. Evers, F.: The academy of the senses: synesthetics in science, art and education. ArtScience Interfaculty Press, The Hague (2012).

24. Kandinsky, W.: Composition VIII. Retrieved 02-11-2016 from https://www.guggenheim.org/artwork/1924 (1924).

25. Ramachandran, V.S. \& Hubbard, E.M. Synaesthesia: a window into perception, thought and language. Journal of Consciousness Studies 8, 12, 3-34 (2001).

26. Maidenbaum, S.: Practical sensory substitution in real and virtual worlds: development, accessibility and neuroscience. In Proceedings of the 33rd Annual ACM Conference Extended Abstracts on Human Factors in Computing Systems, 211-214 (2015).

27. Van Dartel, M., Misker, J., Nigten, A. and Van der Ster, J.: Virtual Reality and Augmented Reality Art Explained in Terms of Sensory-motor Coordination. In Proceedings of 4th International Conference on Enactive Interfaces (2007).

28. Maidenbaum, S., Chebat, D.R., Levy-Tzedek, S., Namer-Furstenberg, R., \& Amedi, A.: The effect of expanded sensory range via the EyeCane sensory substitution device on the characteristics of visionless virtual navigation. Multi-Sensory Research 27, 5-6, 379-397 (2014).

29. Froese, T. \& Spiers, A.: Toward a Phenomenological Pragmatics of Enactive Perception. Cognitive Science Research Papers, 593 (2004).

30. O'Rourke, K.: Walking and Mapping. MIT Press, Cambridge, MA (2013).

31. Studio Eyal Burstein: Eye Candy Can Ltd. Retrieved on 02-11-2016 from http://www.eyalburstein.com/eye-candy/1n5r92f24hltmysrkuxtiuepd6nokx (2007).

32. Wallas, G. The art of thought. Harcourt Brace, New York (1926).

33. Grand Illusions. Reversing goggles. Retrieved 19-04-2017 from http://www.grand-illusions.com/acatalog/Reversing Goggles.html

34. De Rooij, A. \& Jones, S. de Rooij, A. \& Jones, S. Mood and Creativity: An Appraisal Tendency Perspective. In Proceedings of the 9th ACM Conference on Creativity and Cognition, 362-365 (2013). 